\title{
Combining fisetin and ionizing radiation suppresses the growth of mammalian colorectal cancers in xenograft tumor models
}

\author{
JYH-DER LEU ${ }^{1 *}$, BO-SHEN WANG ${ }^{2 *}$, SHU-JUN CHIU ${ }^{3}$, CHUN-YUAN CHANG $^{2}$, \\ CHIEN-CHIH CHEN ${ }^{4}$, FU-DU CHEN $^{5}$, SHIIREVNYAMBA AVIRMED ${ }^{6}$ and YI-JANG LEE ${ }^{2,7}$ \\ ${ }^{1}$ Department of Radiation Oncology, Taipei City Hospital, Renai Branch, Da'an, Taipei 106; \\ ${ }^{2}$ Department of Biomedical Imaging and Radiological Sciences, School of Biomedical Engineering, \\ National Yang-Ming University, Beitou, Taipei 112; ${ }^{3}$ Department of Life Sciences, Tzu Chi University, Hualien 970; \\ ${ }^{4}$ Department of Family Medicine, Taipei City Hospital, Renai Branch, Da'an, Taipei 112; \\ ${ }^{5}$ Department of Electronic Engineering, Hwa-Hsia University of Technology, Zhonghe, Taipei 235, Taiwan, R.O.C.; \\ ${ }^{6}$ Department of Surgery, Mongolian National University of Medical Sciences, Ulaanbaatar 14210, Mongolia; \\ ${ }^{7}$ Biophotonics \& Molecular Imaging Research Center, National Yang-Ming University, Beitou, Taipei 112, Taiwan, R.O.C.
}

Received July 19, 2015; Accepted September 12, 2016

DOI: $10.3892 / \mathrm{ol} .2016 .5345$

\begin{abstract}
Fisetin (3,7,3',4'-tetrahydroxyflavone), which belongs to the flavonoid group of polyphenols and is found in a wide range of plants, has been reported to exhibit a number of biological activities in human cancer cells, including antioxidant, anti-inflammatory, antiangiogenic, anti-invasive and antiproliferative effects. Although previous in vitro studies have shown that fisetin treatment increases the apoptotic rate and enhances the radiosensitivity of human colorectal cancer cells, the in vivo effects of fisetin on tumor growth remain unclear. In the present study a murine xenograft tumor model was employed to investigate the therapeutic effects of fisetin in combination with radiation on CT-26 colon cancer cells and human HCT116 colorectal cancer cells. This revealed that intratumoral injection of fisetin significantly suppressed the growth of CT-26 tumors compared with the untreated control group, but had little effect on the growth of HCT116 tumors. However, fisetin in combination with 2-Gy radiation enhanced tumor suppressor activity in murine colon and human colorectal xenograft tumors, as compared with 2-Gy fractionated radiation administered alone for 5 days and fisetin alone. Interestingly, fisetin downregulated the expression of the oncoprotein securin in a p53-independent manner. However,
\end{abstract}

Correspondence to: Professor Yi-Jang Lee, Department of Biomedical Imaging and Radiological Sciences, School of Biomedical Engineering, National Yang-Ming University, 155, Section 2, Linong Street, Beitou, Taipei 112, Taiwan, R.O.C.

E-mail: yjlee2@ym.edu.tw

${ }^{*}$ Contributed equally

Key words: fisetin, colorectal cancer, radiotherapy, xenograft tumor model, securin securin-null HCT116 tumors showed only moderate sensitivity to fisetin treatment, and the combination of fisetin and radiation did not significantly suppress securin-null HCT116 tumor growth compared with normal HCT116 tumors. Therefore, the role of securin in mediating the effect of fisetin on colorectal cancer growth warrants further investigation. In conclusion, the results of the current study provide important preclinical data for evaluating the efficacy of fisetin and radiation combination treatment as an adjuvant chemoradiotherapy for human colorectal cancers.

\section{Introduction}

Colorectal cancer is the third leading cause of mortality in the Western world (1) and has emerged as a common malignancy in the Asian population as a result of changes in diet and physical activity levels (2). Dietary habits have been related to the risk of colorectal cancer $(3,4)$. Surgery and chemotherapy are the primary treatments for colorectal cancer. Radiotherapy is a typical adjuvant treatment after surgery or chemotherapy for high-stage colorectal cancers $(5,6)$. However, colorectal carcinomas display a wide range of radiosensitivity $(7,8)$. Therefore, new approaches are necessary to enhance the efficacy of radiation treatments for colorectal cancers.

Previous epidemiological studies have shown that the daily inclusion of fruit and vegetables in the diet decreases the risk of colon cancer (9). In addition, it has been reported that flavonoids, which are abundant in numerous plants, protect against a number of tumorigenic processes, including oxidative stress, inflammation, angiogenesis and cell invasion (10-12). Furthermore, flavonoids induce cell cycle arrest, apoptosis and radiosensitivity in cancer cells in vitro (13-15). The flavonoid fisetin (3,7,3',4'-tetrahydroxyflavone) is a polyphenol found in numerous plants. A number of previous reports have shown that fisetin activates p53 activity, and represses the cyclooxygenase-2 and Wnt/epidermal growth factor receptor/nuclear factor-B signaling pathways in human 
cancer cells to promote apoptosis (14,16-18). In addition, fisetin inhibits the spindle checkpoint response that arrests cells in the radiosensitive $\mathrm{G} 2 / \mathrm{M}$ phase $(19,20)$. However, the in vivo effects of fisetin remain unclear. As fisetin is a natural and edible product with acceptable biosafety, the clinical potential of this compound is of particular interest and warrants further investigation.

Securin, which was originally isolated from rat pituitary tumor cells, is alternatively called the pituitary tumor transforming gene (21). Securin is a multi-functional protein that serves a number of biological roles, such as the regulation of cellular transformation, sister chromatid separation $(22,23)$, gene transcription (24) and DNA damage repair $(25,26)$. Notably, securin interacts with p53 and perturbs p53-mediated transcription and apoptosis in tumor cells (22). Thus, securin is regarded as an oncoprotein. The depletion of securin has been reported to sensitize human colorectal cancer cells to various types of treatment, including fisetin, butein and ionizing radiation (27-29). However, whether these effects can be repeated in vivo is unknown.

In the present study, tumor-bearing mice were used to examine the effect of fisetin alone and in combination with radiation on the growth of colorectal tumors in vivo. In addition, tumors with defective securin expression were assessed to investigate if securin depletion would enhance sensitivity to these treatments.

\section{Materials and methods}

Cell culture. Murine CT-26 colon cancer cells, and human HCT116 ${ }^{\mathrm{WT}}$, HCT116 ${ }^{\text {p33-/- }}$, HCT116 $6^{\text {securin-/- }}$ and p53-R273H mutant HT-29 colorectal cancer cell lines (30) were cultured in RPMI-1640 medium (Thermo Fisher Scientific, Inc., Waltham, MA, USA) supplemented with $10 \%$ fetal bovine serum (Thermo Fisher Scientific Inc.), $100 \mathrm{U} / \mathrm{ml}$ penicillin, $100 \mu \mathrm{g} / \mathrm{ml}$ streptomycin and $1 \mathrm{mM}$ sodium pyruvate. Cultures were maintained at $37^{\circ} \mathrm{C}$ in a $95 \%$ humidified incubator (Thermo Fisher Scientific, Inc.) with $5 \% \mathrm{CO}_{2}$ and passaged at $1: 3$ every 2 days.

Mouse xenograft models. A total of 62 male BALB/c nude mice (weight, $20 \mathrm{~g}$; age, 6 weeks) were purchased from the National Laboratory Animal Center (NLAC, Nankang, Taipei, Taiwan). The mice were maintained at $22-24^{\circ} \mathrm{C}$ and $70 \%$ humidity under a 12-h light/dark cycle. Food and water were available ad libitum. Five mice were kept in each $77.4 \times 77.4-\mathrm{cm}$ cage. All animal protocols were approved by the Institutional Animal Care and Use Committee (IACUC) of National Yang-Ming University (Taipei, Taiwan; approval no. 1001270). Prior to tumor xenografting, mice were anesthetized with ketamine (50 mg/kg; IMALGENE ${ }^{\circledR}$; Merial Laboratoire de Toulouse, Lyon, France) and xylazine (15 mg/kg; Sigma-Aldrich; Merck Millipore, Darmstadt, Germany). Subsequently, CT-26 (1x106) or HCT116 $\left(2 \times 10^{6}\right)$ cells were subcutaneously injected into the hind legs of the mice ( $n=5$ for injection of each cell line). Prior to further treatment, mice were maintained until tumors reached $100 \mathrm{~mm}^{3}$. Tumor volume was measured using a caliper and calculated as the following: (Length $\mathrm{x}$ width ${ }^{2}$ ) / 2. Tumor volume was measured every 2-3 days to draw tumor growth curves followed by measurement of body weight.
Reagents and radiation treatments. Fisetin was purchased from Sigma-Aldrich (Sigma-Aldrich) and intratumorally injected at a dose of $5 \mathrm{mg} / \mathrm{kg}$ on days 0 and 7. Tumors were irradiated with $2 \mathrm{~Gy} /$ day for 5 days using an X-ray machine (RS 2000 Biological Research X-ray Irradiator; Rad Source Technologies, Inc., Suwanee, GA, USA) operating at $160 \mathrm{kVp}$ and $25 \mathrm{~mA}$. The dose rate at a source to subject distance of $38 \mathrm{~cm}$ was $1.83 \mathrm{~Gy} / \mathrm{min}$. For combined fisetin and radiation treatment, mice were treated with $5 \mathrm{mg} / \mathrm{kg}$ fisetin followed by 2 Gy irradiation on days 0 and 7 . For the control group, the mice were injected with DMSO at the same volume of dissolved fisetin on days 0 and 7. Five mice were used in each group. These treatments are illustrated on a timeline in Fig. 1. Excluding the tumor site, all areas of the mice were masked using a lead radiation protector.

Western blotting and antibodies. Western blot analysis was performed as described previously (29). The following primary antibodies were used: Anti-p53 (Cat. no. GEX70214; 1:2,000; GeneTex, Inc., Irvine, CA, USA), anti-securin (Cat. no. ab3305; 1:1,000; Abcam, Cambridge, UK) and anti-GAPDH (Cat. no. PB197650; 1:5,000; Thermo Fisher Scientific Inc.). The secondary antibodies included a goat anti-mouse antibody (Cat. no. AP124P; 1:10,000; EMD Millipore, Billerica, MA, USA) for detecting the anti-securin and anti-GAPDH primary antibodies, and a goat anti-rabbit antibody (Cat. no. AP132P; 1:10,000; EMD Millipore) for detecting the anti-p53 primary antibody. Band intensities were measured by densitometry using ImageJ 1.x software (National Institutes of Health, Bethesda, MD, USA) (31).

Cell proliferation measurement. Cells $\left(1 \times 10^{5}\right)$ were seeded into 6-cm culture dishes and collected for hemocytometric calculation every day for 7 days. For each time point, the mean number of cells was calculated from three independent cell cultures. The results were plotted as cell proliferation curves.

Statistical analysis. Results are presented as the mean \pm standard deviation. A Student's t-test was performed to determine if differences between groups were statistically significant. For survival analysis, the Kaplan-Meier estimator was used and the results were analyzed using the log-rank test. Statistical analyses were performed using GraphPad Prism 3.0 software (GraphPad Software, Inc., La Jolla, CA, USA). P<0.05 was considered to indicate a statistically significant difference.

\section{Results}

Fisetin inhibits tumor growth in a mouse CT-26 xenograft model. To examine the effect of fisetin on colorectal cancer growth in vivo, a mouse tumor xenograft model generated by injection of CT-26 colon cancer cells was treated with fisetin. Compared with the untreated control group, a single intratumoral injection of $5 \mathrm{mg} / \mathrm{kg}$ fisetin significantly reduced tumor volume for the following 10 days (Fig. 2A). As tumors appeared to re-grow 6 days after the first injection, a second dose of fisetin was administered on day 11 to examine if tumor growth could be inhibited. The results demonstrated that tumor growth was significantly suppressed for 3 days following the second administration of fisetin, as compared with the control 


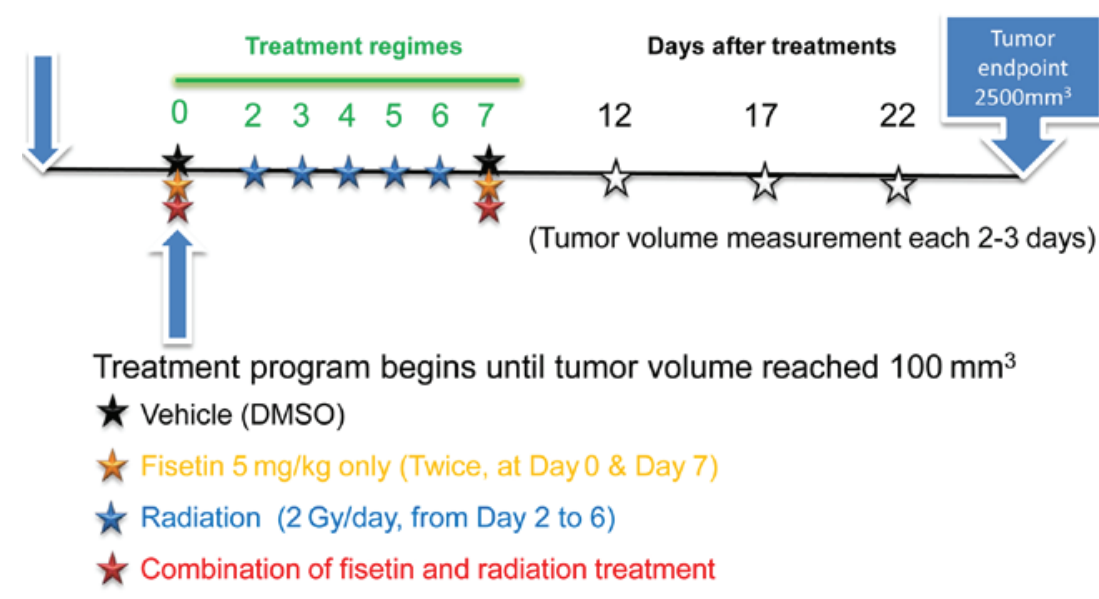

Figure 1. Timeline of treatments of tumor-bearing mice ( $\mathrm{n}=5$ per group). Treatments began when tumor volume reached $100 \mathrm{~mm}^{3}$.

group (Fig. 2A), although tumor growth was not completely inhibited. Fluctuations in body weight between the control and fisetin-treated group were similar (Fig. 2B), indicating that the concentration of fisetin used in this study was not cytotoxic. In addition, the survival rate of fisetin-treated tumor-bearing mice was increased compared with the untreated control group (Fig. 2C). These results demonstrate that administration of fisetin suppresses in vivo tumor growth in a mouse CT-26 xenograft model.

Combining fisetin and radiation for the treatment of mammalian colorectal cancers. Treatment with a combination of fisetin and radiation treatment was investigated in mouse CT-26 and HCT $116^{\text {WT }}$ xenograft tumor models. The timeline of fisetin and radiation treatment is illustrated in Fig. 1; 2-Gy X-rays were administered to the tumor site five times between the first and second fisetin treatments to mimic a clinical regime. The results showed that CT-26 tumor growth was suppressed by fisetin and radiation alone; however, this effect was enhanced by combining the two treatments at 16 to 29 days following treatment (Fig. 3A). Body weight was not significantly different between any of the groups, suggesting that combined fisetin/radiation treatment did not cause systemic toxicity (Fig. 3B). In addition, HCT116 tumor growth was completely and significantly inhibited by combined fisetin/radiation treatment $(\mathrm{P}<0.05$ vs. the control group; Fig. 3C), without significant loss of body weight (Fig. 3D). These results suggest that combined fisetin and radiation treatment is highly effective in suppressing colorectal cancer.

Fisetin induces p53 and suppresses securin protein expression in human colorectal cancer cells. p53 is a tumor suppressor protein that is regulated by a number of different proteins. A previous study demonstrated that the securin oncoprotein binds to p53 and modulates its activity (32). Although securin protein levels have been reported to be elevated by radiation independently of p53 activity (27), it is unknown whether fisetin influences the expression of p53 or securin. In the present study, an experiment with a series of fisetin doses was performed to investigate whether fisetin influences the expression of p53 and securin in different human colorectal cancer cell lines. The results identified that in $\mathrm{HCT} 116^{\mathrm{WT}}$ cells, p53
A

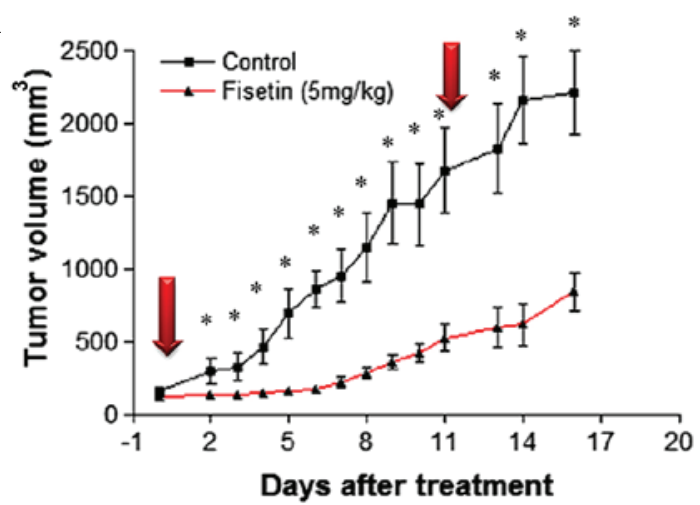

B

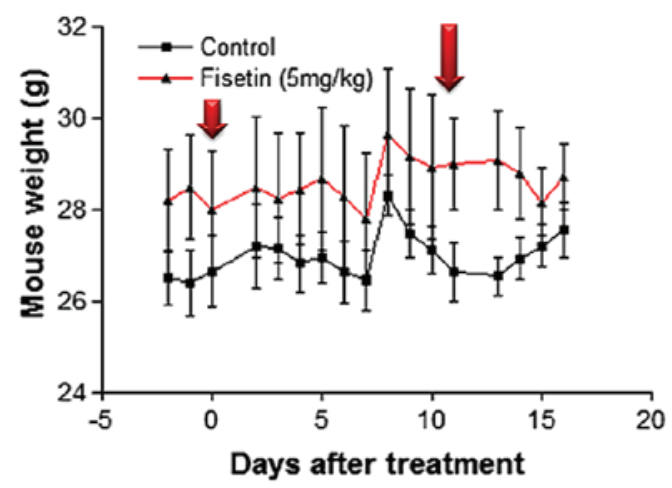

C

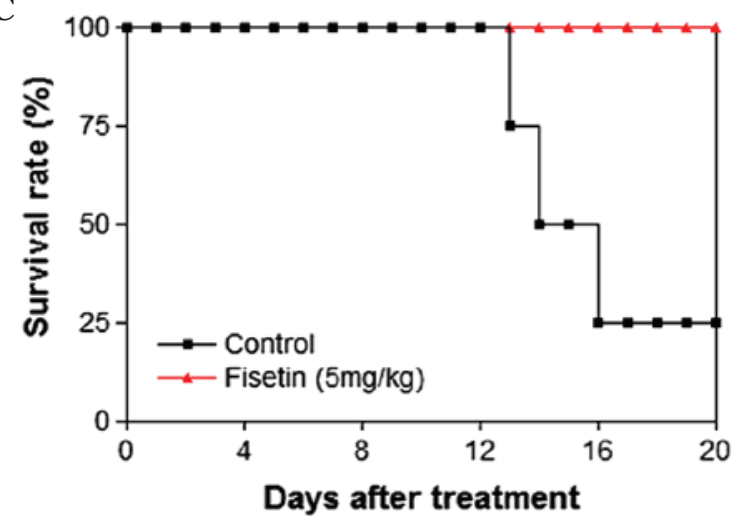

Figure 2. Effect of fisetin on CT-26 colorectal tumors in vivo. (A) Tumor growth curves of tumor-bearing mice with or without intratumoral injection of fisetin ( $\mathrm{n}=5$ for each group). ${ }^{*} \mathrm{P}<0.05$. (B) Body weights of mice following measurement of tumor size. Arrows represent when fisetin injections occurred. (C) Kaplan-Meier estimator survival curves for tumor-bearing mice with or without intratumoral injection of fisetin. 


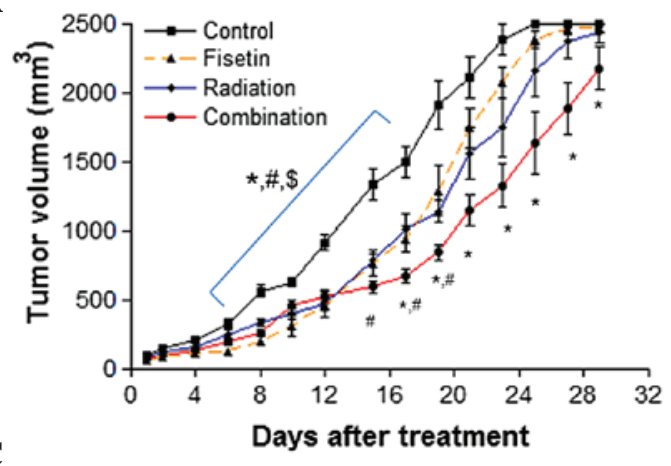

C

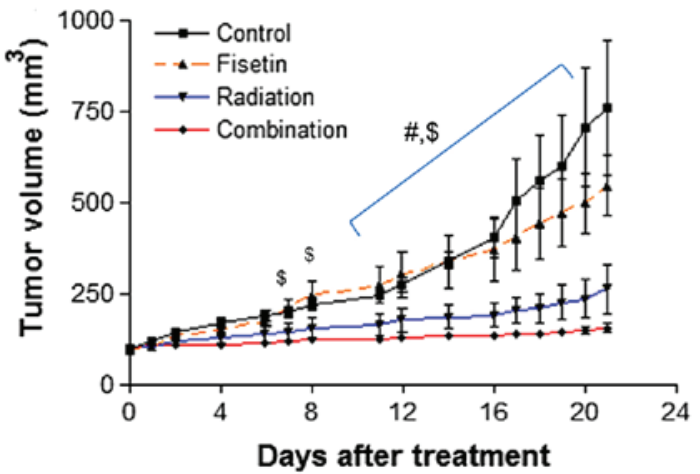

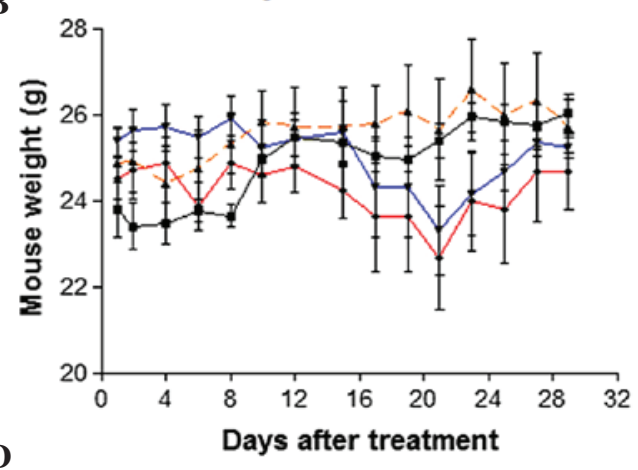

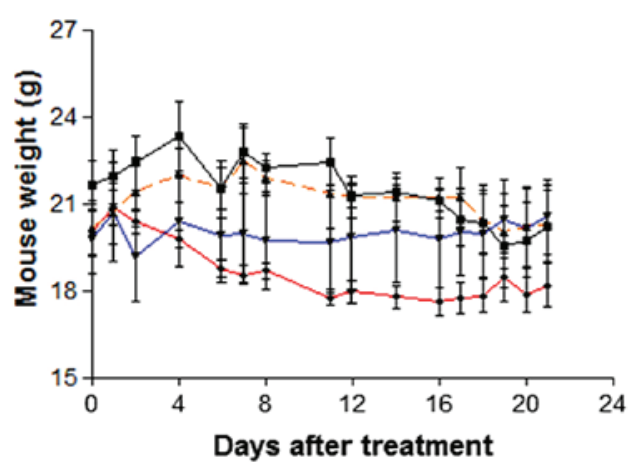

Figure 3. Effect of fisetin and radiation combination treatment on colorectal tumor growth in vivo. (A) Growth curves for CT-26 xenograft tumors. (B) Body weights of mice with CT-26 xenograft tumors. (C) Growth curves for HCT116 xenograft tumors. (D) Body weights of mice with HCT116 xenograft tumors. The symbols *, \# and $\$$ on the top of the black line are $\mathrm{P}<0.05$ for fisetin, radiation and combined treatment vs. the untreated control, respectively; the symbols * and ${ }^{\#}$ beneath the red lines are $\mathrm{P}<0.05$ for fisetin and radiation treatment alone vs. the combined treatment, respectively.

protein levels were increased and securin protein levels were decreased following fisetin treatment (Fig. 4A). Interestingly, fisetin increased the expression of p53 in HCT116 ${ }^{\text {securin-/- }}$ cells and decreased the expression of securin in HCT116 ${ }^{\mathrm{p} 53-/-}$ cells (Fig. 4A). In p53 mutant HT-29 cells, fisetin downregulated the expression of securin protein (Fig. 4A). Quantification of the protein bands on the Western Blot analysis revealed the same results (Fig. 4B). These results indicate that fisetin increases the expression of p53 and decreases the expression of securin, which suppresses tumor growth. Furthermore, fisetin-mediated expression of p53 and securin was independent of the expressive status of p53/securin (null or wild-type).

Effect of fisetin and radiation combination treatment on securin-null colorectal cell-formed xenograft tumors. As fisetin could induce the expression of the p53 protein in the absence of securin, the ability of fisetin and radiation treatment to suppress tumor growth in vivo was investigated. Firstly, the growth curves of HCT116 ${ }^{\text {WT }}$ and HCT116 $6^{\text {securin-- }}$ cells were compared, which showed that the proliferation of HCT116 $16^{\text {securin-- }}$ cells was significantly slower than that of HCT116 ${ }^{\mathrm{WT}}$ cells after 3 days of proliferation (Fig. 5A). Tumor growth was moderately suppressed by fisetin treatment in HCT116 $6^{\text {securin-/- }}$ cells; however, no significant advantage was observed following fisetin and radiation combination treatment (Fig. 5B). The body weight of the mice following fisetin, radiation and fisetin/radiation showed no significant difference compared with the control group (Fig. 5C). Therefore, depletion of securin does not enhance the effects of fisetin and radiation combination treatment on colorectal tumors in vivo.
However, a larger sample size is necessary to validate this result.

\section{Discussion}

Previous studies have shown that fisetin possesses a wide range of activities to suppress the growth of human cancer cells, including breast, prostate, bladder, lung, melanoma and colorectal cancer cells (16,33-39). Among these types of cancer, colorectal cancer is particularly interesting with regards to fisetin, since fisetin is a nutrient supplement that can be administrated orally (40). It is thought that fisetin is absorbed through the digestive tract without the need for intravascular injection (41). Although in vitro studies have demonstrated the efficacy of fisetin in the treatment of colorectal cancer $(15,16)$, few in vivo studies have been reported. Since preclinical studies are essential for potent therapeutic agents to be accepted for clinical trials, the experimental animal data in the present study is important for evaluating the application of fisetin to colorectal cancer therapy. In addition, the current study investigated whether fisetin treatment combined with ionizing radiation exerted synergistic effects. A previous study reported that a combination of cisplatin and fisetin exerted anticancer activity in embryonic carcinoma cells in vitro and in vivo (42). Although radiotherapy is not typically used in the treatment of colorectal cancer, it is frequently applied to adjuvant chemotherapy in rectal cancer $(43,44)$. The combination of fisetin and radiotherapy is an interesting alternative adjuvant therapy, since it would likely avoid the side effects associated with chemotherapy. 
A
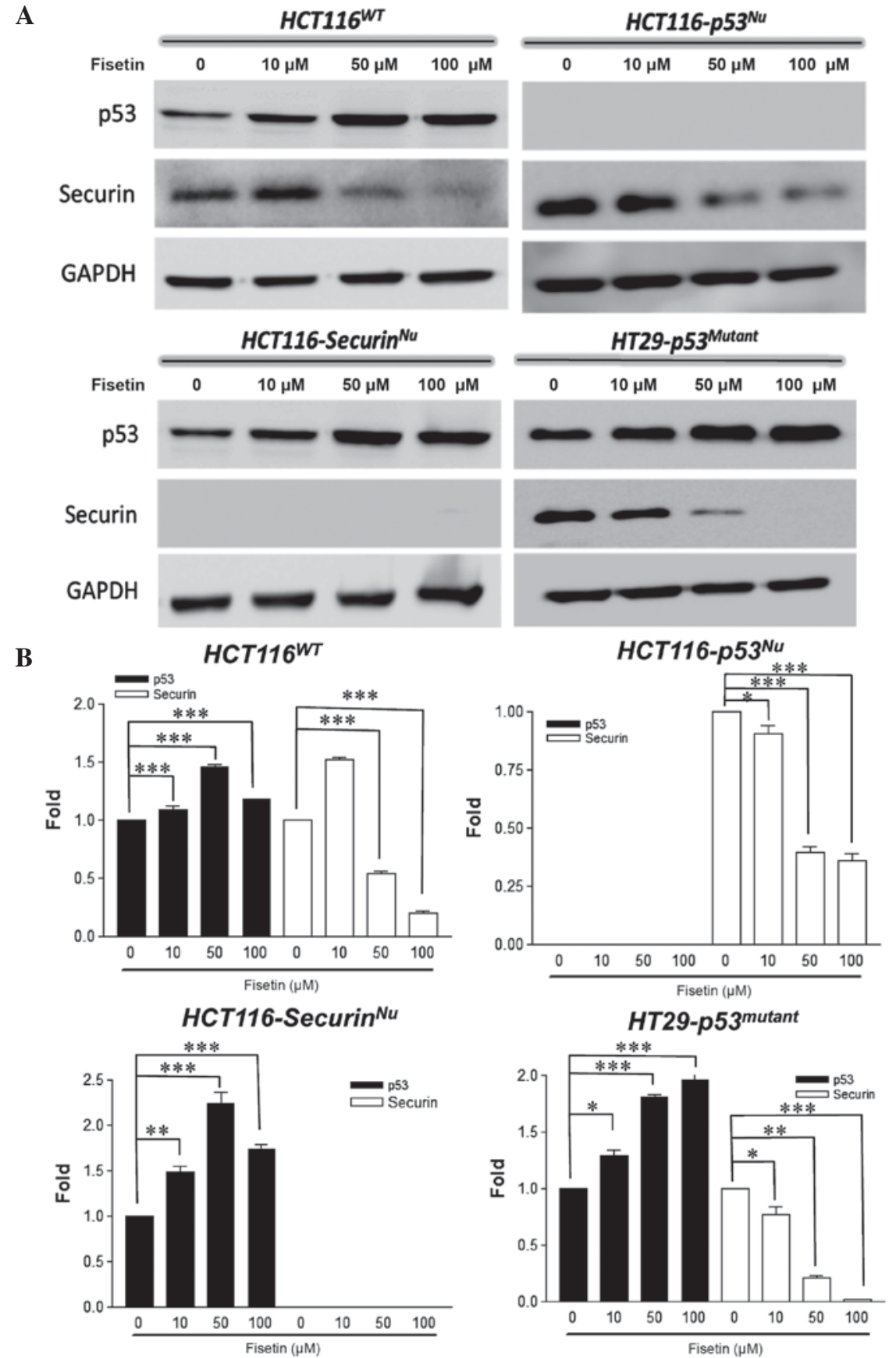

Figure 4. Effect of fisetin on p53 and securin expression in human colorectal cancer cells. (A) Western blots detecting the expression of p53 and securin in wild-type, p53-null and securin-null HCT116 cells, and p53 mutant HT-29 cells, following treatment with different concentrations of fisetin. (B) Quantification of western blots. ${ }^{*} \mathrm{P}<0.05,{ }^{* *} \mathrm{P}<0.0005$ and ${ }^{* * *} \mathrm{P}<0.0001$ vs. $0 \mu \mathrm{M}$ fisetin.

In the present study, a mouse xenograft model was used to examine the tumor-suppressive efficacy of fisetin in vivo on colon and colorectal cancers. Animal (CT-26) and human (HCT116) colon and colorectal cancer cells, respectively, were used to establish xenograft tumor models. Fisetin treatment was shown to suppress the growth of tumors formed by CT-26 cells, but not HCT116 cells. However, treatment with fisetin combined with radiation exhibited enhanced tumor suppressive effects on CT-26 and HCT116 xenograft tumors. This finding supports the potential application of fisetin to adjuvant radiotherapy for colorectal cancers. As fisetin did not exert toxicity in the current study, or in other reports $(45,46)$, its use as an adjuvant treatment should be feasible.
In the present study, CT-26 and HCT116 cell lines, which express wild-type p53, formed tumors that were sensitive to radiation treatment. Since p53 is known to be upregulated by radiation (47), it is reasonable that this phenomenon was observed in vivo in the present study. Importantly, the current study used clinically comparable fractionated radiation of 2-Gy/day/fraction for 5 days (48). Compared with this regime, the fisetin-combined radiation treatments only irradiated tumors at 2 Gy twice in one week, yet remained more efficient than the fractionated radiation on tumor suppression, suggesting that fisetin enhances the tumor response to radiation in vivo. Thus, the use of fisetin in adjuvant radiotherapy may reduce total radiation exposure and improve the quality of life of patients during 
A

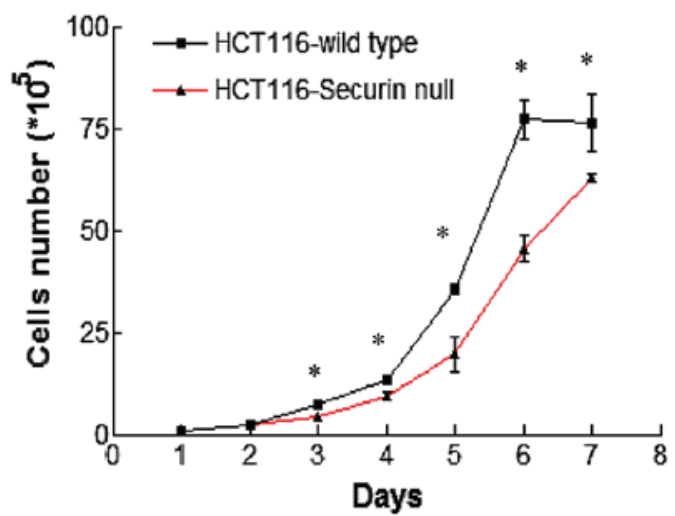

B

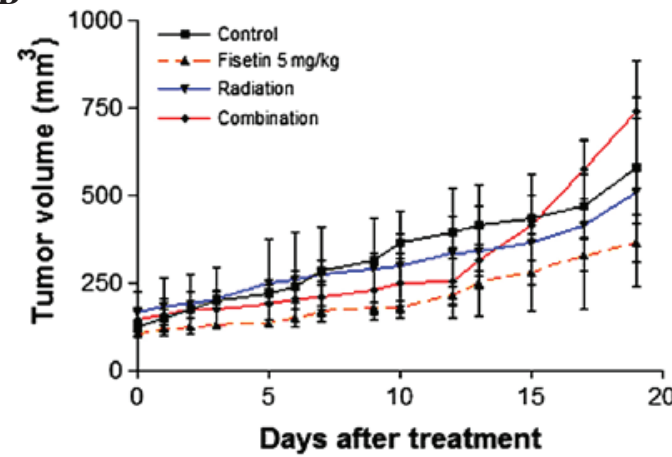

C

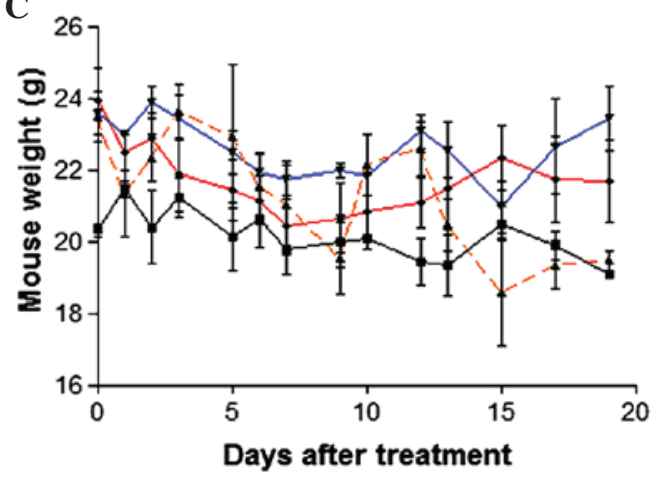

Figure 5. Effect of fisetin and radiation combination treatment on the growth of securin-null HCT116 xenograft tumors in vivo. (A) Cell growth curves of HCT116 ${ }^{\mathrm{WT}}$ cells and HCT116 ${ }^{\text {securin-/- }}$ cells. The cell number of each time point was compared. "P<0.05 vs. the same time point in the HCT116 ${ }^{\text {securin-/- }}$ cells. (B) Growth curves of HCT116 $6^{\text {securin-/ }}$ xenograft tumors following treatment with fisetin, radiation, or fisetin and radiation combined ( $\mathrm{n}=3$ per group). (C) Body weights of mice with HCT116 $6^{\text {securin-/ }}$ xenograft tumors.

and following treatment. Further investigations into the optimal combination for colorectal cancer therapy are warranted.

The genetic background of a tumor is known to influence the prognosis $(49,50)$. The authors of the present study were interested in p53 and securin because of the results of our previous studies $(15,27)$. Briefly, fisetin was shown to enhance the radiosensitivity of p53-mutant human HT-29 colorectal cancer cells, and promote apoptosis in securin-depleted human HCT-116 colorectal cancer cells. Consistent with a previous report (14), in the present study, we also showed that fisetin could induce p53 protein expression in wild-type and securin-null HCT116 cells. Furthermore, expression of the securin protein was downregulated by fisetin regardless of the p53 expression status. In addition, in the current study xenograft securin-null colorectal tumors exhibited increased sensitivity to fisetin compared with the untreated control. However, individual variance in the tumor-bearing mice may have reduced the extent of tumor suppression resulting from fisetin treatment. Therefore, an increased sample size is required to validate the effect of fisetin on securin-null colorectal cancers. In addition, this limitation should be considered for the investigation of fisetin and radiation combination treatment. In the current study, p53 protein expression was still induced in securin-null HCT116 cells by fisetin, indicating that p53 and securin protein expression should be modulated by fisetin and radiation combination treatment in order to achieve optimal tumor suppression.

In conclusion, the present study used mouse xenograft tumor models to investigate the effect of fisetin alone or in combination with radiation on colon and colorectal tumor growth. The results showed that fisetin treatment alone was sufficient to suppress murine CT-26 colon tumors, but not human HCT116 colorectal tumors. Interestingly, a combination of fisetin and radiation treatment enhanced the tumor suppression compared with fractionated irradiation alone. In addition, the results of the current study revealed that securin-null HCT116 tumors exhibited increased sensitivity to fisetin treatment, although this observation needs to be validated in a larger sample set. Investigating whether securin is the key molecule in mediating the effects of fisetin and radiation combination treatment on colon and colorectal cancers warrants future exploration. To the best of our knowledge, this is the first report demonstrating the therapeutic efficacy of fisetin and radiation combination treatment on colon and colorectal cancer in vivo. The results of the present study provide important preclinical information for evaluating the potential use of fisetin in adjuvant cancer radiotherapy.

\section{Acknowledgements}

The present study was supported by the Taipei City Government Department of Health (Taipei, Taiwan; grant no. 10101-62-034) and the Ministry of Science and Technology (Taipei, Taiwan; grant nos. 102-2628-B-010-012-MY3 and 105-2628-B-010-013-MY3).

\section{References}

1. Siegel R, Desantis C and Jemal A: Colorectal cancer statistics, 2014. CA Cancer J Clin 64: 104-117, 2014. 
2. Davies NJ, Batehup L and Thomas R: The role of diet and physical activity in breast, colorectal, and prostate cancer survivorship: A review of the literature. Br J Cancer 105: (Suppl 1) S52-S73, 2011.

3. Doll R and Peto R: The causes of cancer: Quantitative estimates of avoidable risks of cancer in the United States today. J Natl Cancer Inst 66: 1191-1308, 1981.

4. Lin J, Zhang SM, Cook NR, Rexrode KM, Liu S, Manson JE, Lee IM and Buring JE: Dietary intakes of fruit, vegetables, and fiber, and risk of colorectal cancer in a prospective cohort of women (United States). Cancer Causes Control 16: 225-233, 2005.

5. Hamaya Y, Guarinos C, Tseng-Rogenski SS, Iwaizumi M, Das R, Jover R, Castells A, Llor X, Andreu M and Carethers JM: Efficacy of Adjuvant 5-Fluorouracil Therapy for Patients with EMAST-Positive Stage II/III Colorectal Cancer. PLoS One 10: e0127591, 2015.

6. Saltz LB and Minsky B: Adjuvant therapy of cancers of the colon and rectum. Surg Clin North Am 82: 1035-1058, 2002.

7. Allal AS, Kähne T, Reverdin AK, Lippert H, Schlegel W and Reymond MA: Radioresistance-related proteins in rectal cancer. Proteomics 4: 2261-2269, 2004.

8. Ma W, Yu J, Qi X, Liang L, Zhang Y, Ding Y, Lin X, Li G and Ding Y: Radiation-induced microRNA-622 causes radioresistance in colorectal cancer cells by down-regulating $\mathrm{Rb}$. Oncotarget 6: 15984-15994, 2015.

9. Lock K, Pomerleau J, Causer L, Altmann DR and McKee M: The global burden of disease attributable to low consumption of fruit and vegetables: Implications for the global strategy on diet. Bull World Health Organ 83: 100-108, 2005.

10. Martinez-Perez C, Ward C, Cook G, Mullen P, McPhail D, Harrison DJ and Langdon SP: Novel flavonoids as anti-cancer agents: Mechanisms of action and promise for their potential application in breast cancer. Biochem Soc Trans 42: 1017-1023, 2014.

11. Orlikova B, Menezes JC, Ji S, Kamat SP, Cavaleiro JA and Diederich M: Methylenedioxy flavonoids: Assessment of cytotoxic and anti-cancer potential in human leukemia cells. Eur J Med Chem 84: 173-180, 2014.

12. Imai M, Kikuchi H, Denda T, Ohyama K, Hirobe $C$ and Toyoda $H$ : Cytotoxic effects of flavonoids against a human colon cancer derived cell line, COLO 201: A potential natural anti-cancer substance. Cancer Lett 276: 74-80, 2009.

13. Lu X, Jung JI, Cho HJ, Lim DY, Lee HS, Chun HS, Kwon DY and Park JH: Fisetin inhibits the activities of cyclin-dependent kinases leading to cell cycle arrest in HT-29 human colon cancer cells. J Nutr 135: 2884-2890, 2005.

14. Lim DY and Park JH: Induction of p53 contributes to apoptosis of HCT-116 human colon cancer cells induced by the dietary compound fisetin. Am J Physiol Gastrointest Liver Physiol 296 : G1060-G1068, 2009.

15. Chen WS, Lee YJ, Yu YC, Hsaio CH, Yen JH, Yu SH, Tsai YJ and Chiu SJ: Enhancement of p53-mutant human colorectal cancer cells radiosensitivity by flavonoid fisetin. Int J Radiat Oncol Biol Phys 77: 1527-1535, 2010.

16. Suh Y, Afaq F, Johnson JJ and Mukhtar H: A plant flavonoid fisetin induces apoptosis in colon cancer cells by inhibition of COX2 and Wnt/EGFR/NF-kappaB-signaling pathways. Carcinogenesis 30: 300-307, 2009.

17. Li J, Cheng Y, Qu W, Sun Y, Wang Z, Wang H and Tian B: Fisetin, a dietary flavonoid, induces cell cycle arrest and apoptosis through activation of p53 and inhibition of NF-kappa B pathways in bladder cancer cells. Basic Clin Pharmacol Toxicol 108: 84-93, 2011.

18. Chou RH, Hsieh SC, Yu YL, Huang MH, Huang YC and Hsieh YH: Fisetin inhibits migration and invasion of human cervical cancer cells by down-regulating urokinase plasminogen activator expression through suppressing the $\mathrm{p} 38 \mathrm{MAPK}$-dependent NF- $\mathrm{KB}$ signaling pathway. PloS one 8: e71983, 2013.

19. Salmela AL, Pouwels J, Varis A, Kukkonen AM, Toivonen P, Halonen PK, Perälä M, Kallioniemi O, Gorbsky GJ and Kallio MJ Dietary flavonoid fisetin induces a forced exit from mitosis by targeting the mitotic spindle checkpoint. Carcinogenesis 30: 1032-1040, 2009.

20. Haddad AQ, Venkateswaran V, Viswanathan L, Teahan SJ, Fleshner NE and Klotz LH: Novel antiproliferative flavonoids induce cell cycle arrest in human prostate cancer cell lines. Prostate Cancer Prostatic Dis 9: 68-76, 2006.

21. Pei L and Melmed S: Isolation and characterization of a pituitary tumor-transforming gene (PTTG). Mol Endocrinol 11: 433-441, 1997.

22. RustgiAK: Securinanew roleforitself.NatGenet32:222-224,2002.
23. Zou H, McGarry TJ, Bernal T and Kirschner MW: Identification of a vertebrate sister-chromatid separation inhibitor involved in transformation and tumorigenesis. Science 285: 418-422, 1999.

24. Tong $\mathrm{Y}$ and Eigler T: Transcriptional targets for pituitary tumor-transforming gene-1. J Mol Endocrinol 43: 179-185, 2009.

25. Kim DS, Franklyn JA, Smith VE, Stratford AL, Pemberton HN Warfield A, Watkinson JC, Ishmail T, Wakelam MJ and McCabe CJ: Securin induces genetic instability in colorectal cancer by inhibiting double-stranded DNA repair activity. Carcinogenesis 28: 749-759, 2007.

26. Nagao K, Adachi Y and Yanagida M: Separase-mediated cleavage of cohesin at interphase is required for DNA repair. Nature 430: 1044-1048, 2004

27. Yu SH, Yang PM, Peng CW, Yu YC and Chiu SJ: Securin depletion sensitizes human colon cancer cells to fisetin-induced apoptosis. Cancer Lett 300: 96-104, 2011.

28. Huang YT, Lin CI, Chien PH, Tang TT, Lin J and Chao JI: The depletion of securin enhances butein-induced apoptosis and tumor inhibition in human colorectal cancer. Chem Biol Interact 220: 41-50, 2014.

29. Chen WS, Yu YC, Lee YJ, Chen JH, Hsu HY and Chiu SJ: Depletion of securin induces senescence after irradiation and enhances radiosensitivity in human cancer cells regardless of functional p53 expression. Int J Radiat Oncol Biol Phys 77: 566-574, 2010

30. Haupt S, di Agostino S, Mizrahi I, Alsheich-Bartok O, Voorhoeve M, Damalas A, Blandino G and Haupt Y: Promyelocytic leukemia protein is required for gain of function by mutant p53. Cancer Res 69: 4818-4826, 2009.

31. Schneider CA, Rasband WS and Eliceiri KW: NIH Image to ImageJ: 25 years of image analysis. Nat Methods 9: 671-675, 2012.

32. Bernal JA, Luna R, Espina A, Lázaro I, Ramos-Morales F, Romero F, Arias C, Silva A, Tortolero M and Pintor-Toro JA: Human securin interacts with p53 and modulates p53-mediated transcriptional activity and apoptosis. Nat Genet 32: 306-311, 2002.

33. Yang PM, Tseng HH, Peng CW, Chen WS and Chiu SJ: Dietary flavonoid fisetin targets caspase-3-deficient human breast cancer MCF-7 cells by induction of caspase-7-associated apoptosis and inhibition of autophagy. Int J Oncol 40: 469-478, 2012

34. Khan N, Afaq F, Syed DN and Mukhtar H: Fisetin, a novel dietary flavonoid, causes apoptosis and cell cycle arrest in human prostate cancer LNCaP cells. Carcinogenesis 29: 1049-1056, 2008

35. Li J, Qu W, Cheng Y, Sun Y, Jiang Y, Zou T, Wang Z, Xu Y and Zhao H: The inhibitory effect of intravesical fisetin against bladder cancer by induction of p53 and down-regulation of NF-kappa B pathways in a rat bladder carcinogenesis model. Basic Clin Pharmacol Toxicol 115: 321-329, 2014.

36. Kang KA, Piao MJ and Hyun JW: Fisetin induces apoptosis in human nonsmall lung cancer cells via a mitochondria-mediated pathway. In Vitro Cell Dev Biol Anim 51: 300-309, 2015.

37. Syed DN, Chamcheu JC, Khan MI, Sechi M, Lall RK, Adhami VM and Mukhtar H: Fisetin inhibits human melanoma cell growth through direct binding to p70S6K and mTOR: Findings from 3-D melanoma skin equivalents and computational modeling. Biochem Pharmacol 89: 349-360, 2014.

38. Pal HC, Sharma S, Strickland LR, Katiyar SK, Ballestas ME, Athar M, Elmets CA and Afaq F: Fisetin inhibits human melanoma cell invasion through promotion of mesenchymal to epithelial transition and by targeting MAPK and $\mathrm{NF \kappa B}$ signaling pathways. PloS one 9: e86338, 2014.

39. Szliszka E, Helewski KJ, Mizgala E and Krol W: The dietary flavonol fisetin enhances the apoptosis-inducing potential of TRAIL in prostate cancer cells. Int J Oncol 39: 771-779, 2011

40. Khan N, Syed DN, Ahmad N and Mukhtar H: Fisetin: A dietary antioxidant for health promotion. Antioxid Redox Signal 19: 151-162, 2013

41. Sahu BD, Kumar JM and Sistla R: Fisetin, a dietary flavonoid, ameliorates experimental colitis in mice: Relevance of NF- $\kappa B$ signaling. J Nutr Biochem 28: 171-182, 2016.

42. Tripathi R, Samadder T, Gupta S, Surolia A and Shaha C: Anticancer activity of a combination of cisplatin and fisetin in embryonal carcinoma cells and xenograft tumors. Mol Cancer Ther 10: 255-268, 2011. 
43. Breugom AJ, van Gijn W, Muller EW, Berglund $\AA$, van den Broek CB, Fokstuen T, Gelderblom H, Kapiteijn E, Leer JW, Marijnen CA, et al: Adjuvant chemotherapy for rectal cancer patients treated with preoperative (chemo)radiotherapy and total mesorectal excision: A dutch colorectal cancer group (DCCG) randomized phase III trial. Ann Oncol 26: 696-701, 2015.

44. Colorectal Cancer Collaborative Group: Adjuvant radiotherapy for rectal cancer: A systematic overview of 8,507 patients from 22 randomised trials. Lancet 358: 1291-1304, 2001.

45. Currais A, Prior M, Dargusch R, Armando A, Ehren J, Schubert D, Quehenberger O and Maher P: Modulation of p25 and inflammatory pathways by fisetin maintains cognitive function in Alzheimer's disease transgenic mice. Aging Cell 13: 379-390, 2014.
46. da Costa MP, Bozinis MC, Andrade WM, Costa CR, da Silva AL, Alves de Oliveira CM, Kato L, Fernandes Ode F, Souza LK and Silva Mdo R: Antifungal and cytotoxicity activities of the fresh xylem sap of Hymenaea courbaril L. and its major constituent fisetin. BMC Complement Altern Med 14: 245, 2014.

47. Fei P and El-Deiry WS: P53 and radiation responses. Oncogene 22: 5774-5783, 2003.

48. Fertil B and Malaise EP: Inherent cellular radiosensitivity as a basic concept for human tumor radiotherapy. Int J Radiat Oncol Biol Phys 7: 621-629, 1981.

49. Lindström LS, Hall P, Hartman M, Wiklund F and Czene K: Is genetic background important in lung cancer survival? PloS one 4: e5588, 2009.

50. Ashworth A, Lord CJ and Reis-Filho JS: Genetic interactions in cancer progression and treatment. Cell 145: 30-38, 2011 